\title{
Characteristics of weak base-induced vacuoles formed around individual acidic organelles
}

\author{
Hiromi Hiruma, Tadashi Kawakami
}

Department of Physiology, Kitasato University School of Medicine, Kitasato, Sagamihara, Japan

\begin{abstract}
We have previously found that the weak base 4-aminopyridine induces Brownian motion of acidic organelles around which vacuoles are formed, causing organelle traffic disorder in neurons. Our present study investigated the characteristics of vacuoles induced by weak bases $\left(\mathrm{NH}_{4} \mathrm{Cl}\right.$, aminopyridines, and chloroquine) using mouse cells. Individual vacuoles included acidic organelles identified by fluorescent protein expression. Mitochondria and actin filaments were extruded outside the vacuoles, composing the vacuole rim. Staining with amine-reactive fluorescence showed no protein/amino acid content in vacuoles. Thus, serous vacuolar contents are probably partitioned by viscous cytosol, other organelles, and cytoskeletons, but not membrane. The weak base (chloroquine) was immunochemically detected in intravacuolar organelles, but not in vacuoles. Early vacuolization was reversible, but long-term vacuolization caused cell death. The vacuolization and cell death were blocked by the vacuolar $\mathrm{H}^{+}$-ATPase inhibitor and $\mathrm{Cl}^{-}$-free medium. Staining with LysoTracker or LysoSensor indicated that intravacuolar organelles were strongly acidic and vacuoles were slightly acidic. This suggests that vacuolization is caused by accumulation of weak base and $\mathrm{H}^{+}$in acidic organelles, driven by vacuolar $\mathrm{H}^{+}$-ATPase associated with $\mathrm{Cl}^{-}$entering, and probably by subsequent extrusion of $\mathrm{H}^{+}$and water from organelles to the surrounding cytoplasm. (Folia Histochemica et Cytobiologica 2011; Vol. 49, No. 2, pp. 272-279)
\end{abstract}

Key words: acidic organelles, cytoplasmic vacuoles, vacuolar $\mathrm{H}^{+}$-ATPase, weak base

\section{Introduction}

Vacuolization of the cytoplasm in mammalian cells is a sign of serious cell damage. Indeed, vacuolated cells are frequently observed in various pathological conditions such as wasting diseases, degenerative diseases, infections, and poisoning [1-4]. Experimentally, cytoplasmic vacuoles can be produced by treating cells with some membrane-permeable weak bases [5], because these weak bases are preferentially internalized into acidic organelles such as lysosomes. A similar internalization mechanism is suggested in the formation of vacuoles induced by toxins of Helicobacter pylori [1] and Vibrio cholerae [4]. How are vacuoles formed subsequent to internalization of

Correspondence address: H. Hiruma, Department of Physiology, Kitasato University School of Medicine 1-15-1 Kitasato, Minami-ku, Sagamihara, 252-0374 Japan; tel.: (+ 81 42) 77891 59, fax: (+ 81 42) 77898 41;

e-mail:hiruma@med.kitasato-u.ac.jp weak bases or toxins? One theory proposes that osmotically swollen lysosomes are vacuoles themselves, on the basis of the idea that a high concentration of weak bases accumulated in the lysosomes may take up water through the lysosomal membrane $[5,6]$. Another theory suggests that the weak base-induced vacuoles are autophagic vacuoles, since vacuoles contain cytoplasmic membranous structure [7]. Recently, we found that the weak base 4-aminopyridine induces Brownian movement of acidic organelles and vacuoles are formed around individual acidic organelles, causing organelle traffic disorder in cultured dorsal root ganglion (DRG) neurons [8]. In the present study, the characteristics of vacuoles induced by the known vacuole-forming weak bases $\mathrm{NH}_{4} \mathrm{Cl}$, aminopyridines, and chloroquine were investigated in mouse neuronal and non-neuronal cells to clarify the vacuolization process and mechanism. Aminopyridines are widely used as a membrane-permeable $\mathrm{K}^{+}$ channel blocker and are known to release neurotransmitters $[9,10]$. These agents are now used in the treat- 
ment of multiple sclerosis [11]. Chloroquine is a well-known anti-malaria drug.

\section{Material and methods}

Cell culture. The experimental protocol was approved by the Animal Experimentation and Ethics Committee of Kitasato University School of Medicine. Neuronal and non-neuronal cells were obtained from mouse dorsal root ganglia. Adult male C57BL/6 mice (aged eight weeks) were euthanized with ether, and the dorsal root ganglia were removed and incubated at $37^{\circ} \mathrm{C}$ for 90 minutes with $2 \mathrm{mg} / \mathrm{ml}$ collagenase, and subsequently for 15 minutes with $2.5 \mathrm{mg} /$ $\mathrm{ml}$ trypsin. Then, the ganglia were triturated using fire-polished pipettes (inner diameter: $0.5 \mathrm{~mm}$ ). The cells were plated onto polylysine-coated coverslips and cultured for 48 hours at $37^{\circ} \mathrm{C}$ under $5 \% \mathrm{CO}_{2}$ (pH 7.4) in Ham's F-12 medium containing $10 \%$ fetal bovine serum and penicillin $(60 \mu \mathrm{g} / \mathrm{ml})$-streptomycin $(100 \mu \mathrm{g} / \mathrm{ml})$. Cells were used for experiments after being cultured for 48-72 hours. At that time, some DRG neurons exhibited neurites and some nonneuronal cells were proliferating.

Pharmacological manipulations. The composition of physiological salt solution was $135 \mathrm{mM} \mathrm{NaCl}, 5 \mathrm{mM} \mathrm{KCl}, 1 \mathrm{mM}$ $\mathrm{CaCl}_{2}, 1 \mathrm{mM} \mathrm{MgCl}_{2}, 10 \mathrm{mM}$ HEPES, and $5.5 \mathrm{mM}$ glucose. Chloride $\left(\mathrm{Cl}^{-}\right)$-free solution was composed of $67.5 \mathrm{mM}$ $\mathrm{Na}_{2} \mathrm{SO}_{4}, 2.5 \mathrm{mM} \mathrm{K}_{2} \mathrm{SO}_{4}, 1 \mathrm{mM} \mathrm{CaSO}, 1 \mathrm{mM} \mathrm{MgSO}$, $10 \mathrm{mM}$ HEPES, and $5.5 \mathrm{mM}$ glucose. The osmolarity of both solutions was $285 \pm 5 \mathrm{mosmol} / \mathrm{kg}$. Ammonium chloride $\left(\mathrm{NH}_{4} \mathrm{Cl}, 10 \mathrm{mM}\right.$, Wako, Osaka, Japan), chloroquine diphosphate salt (10 $\mu \mathrm{M}$, Sigma-Aldrich, St Louis, MO, USA), and aminopyridines (4 mM) [2-aminopyridine (Wako), 3-aminopyridine (Wako), 4-aminopyridine (Wako), and 3,4-diaminopyridine (Sigma-Aldrich)] were dissolved directly in physiological salt solutions. The vacuolar $\mathrm{H}^{+}$-ATPase inhibitor bafilomycin A1 (Wako) was dissolved in dimethyl sulfoxide (DMSO) (Sigma-Aldrich) and then diluted with physiological salt solution at a concentration of $1 \mu \mathrm{M}$. The final DMSO concentration was $0.1 \%$ and this concentration of DMSO had no effect on cell vacuolization. In all solutions, $\mathrm{pH}$ was adjusted to 7.4. All biological experiments were performed at room temperature $\left(24-25^{\circ} \mathrm{C}\right)$.

Video-microscopic observation. The coverslip on which cells were cultured was attached with waterproof tape to the underside of a thin stainless steel plate $(0.5-\mathrm{mm}$ thickness) with a hollowed out hexagon. The topside of the plate was covered with another coverslip, leaving spaces at two opposite angles of the hexagon for drug injection. Then, the culture medium was replaced with a physiological salt solution. The chamber was placed on the stage of an inverted Zeiss Axiomat microscope with an oil-immersed PlanApochromat $63 \times$ objective (Carl Zeiss, Oberkochen, Germany). Differential interference contrast (DIC) images obtained by the microscope were transformed into video signals by a video camera (Harpicon, Hamamatsu Photonics, Hamamatsu, Japan) and a camera controller (C2741, Hamamatsu Photonics). The signals were processed to digital video images with enhanced contrast by a video image enhancement system (DVS-20, Hamamatsu Photonics). Video images of cells before, during, and after treatment with drugs were displayed in real-time on a video monitor (C1864, Hamamatsu Photonics) and stored on a video recorder (PVW-2800, Sony, Tokyo, Japan).

Fluorescent protein labeling. Lysosomes, mitochondria, and actin filaments were labeled with fluorescent proteins using baculovirus system, Organelle Lights ${ }^{\mathrm{TM}}$ and Cellular Lights $^{\mathrm{TM}}$ reagents (Invitrogen, Calsbad, CA, USA). These are baculovirus suspensions carrying an expression construct that produces a fluorescent protein fused to cellular proteins. Twenty hours after culture, the cells were incubated, gently rotating with Organelle Lights ${ }^{\mathrm{TM}}$ mito-GFP (for labeling mitochondria with green fluorescent protein, GFP), Organelle Lights ${ }^{\mathrm{TM}}$ lyso-RFP (for labeling lysosomes with red fluorescent protein, RFP), or Cellular Lights ${ }^{\mathrm{TM}}$ actin-GFP (for labeling actin filaments with GFP) diluted with Dulbecco's phosphate-buffered saline (D-PBS) (1:1). After a two hour incubation, the reagents were removed and washed with D-PBS. Cells were then incubated in culture medium for 48 hours. At that time, approximately $10 \%$ of non-neuronal cells expressed fluorescent proteins. Images of organelles and actin filaments visualized by fluorescent proteins were obtained by confocal microscope before and after a one hour treatment of cells with weak base.

Chemical fluorescent labeling. Cells were treated for one hour with weak base. Control cells were treated with physiological salt solution alone. Subsequently, in order to label cellular proteins and amino acids, cells were stained for five minutes with 5-(and -6)-carboxy 2',7'-dichlorofluorescein diacetate succinimidyl ester (CFDA-SE; $1 \mu \mathrm{M}$; Invitrogen), which was dissolved in DMSO and then diluted 1:10,000 with physiological salt solution, and finally washed with physiological salt solution. In order to determine the $\mathrm{H}^{+}$distribution in the cell, each of the low $\mathrm{pH}$ indicators, LysoTracker® Green (1:1,000; Invitrogen) and LysoSensor ${ }^{\mathrm{TM}}$ Yellow/ /Blue (1:200, Invitrogen), diluted with physiological salt solution, was applied for five minutes to the cells and then washed with physiological salt solution. Cells pre-loaded with the $\mathrm{pH}$ indicators, and then treated with the weak base, were also used.

Immunocytochemistry. Immunocytochemistry was performed on fixed cells. Cells after treatment with weak base for one hour were fixed with $4 \%$ paraformaldehyde for 20 minutes and washed for ten minutes with phosphate-buffered saline (PBS) containing 0.3\% Triton X-100 (PBST), and then treated for ten minutes with protein blocking agent 
(Immunon $^{\mathrm{TM}}$; Shandon, Pittsburgh, PA, USA) to block non-specific protein sites. The cells were incubated for one hour with an antibody, rat monoclonal anti-mouse cathepsin D (1:200; R\&D Systems, Minneapolis, MN, USA) or mouse monoclonal anti-chloroquine (1:200; Antibodyshop, Gentofte, Denmark). The antibodies were diluted with $0.2 \%$ bovine serum albumin, $1 \%$ normal goat serum, and $0.1 \%$ sodium azide in PBST. After washing with PBS, the cells were incubated for one hour with Alexa fluor 488-conjugated donkey anti-rat IgG (1:200; Invitrogen) for cathepsin D or Alexa fluor 488-conjugated goat anti-mouse IgG (1:200; Invitrogen) for chloroquine. Finally, the cells were washed with PBS.

Confocal and fluorescence microscopic observations. The cells stained with fluorescence were examined using a Zeiss LSM510 confocal microscope equipped with argon (488 nm) and helium-neon $(543 \mathrm{~nm})$ lasers to excite green and red fluorescence, respectively. A $100 \times / 1.4$ oil immersion objective was used. The cells stained with LysoSensor Yellow/ /Blue were examined by an Axiovert 135 TV fluorescence microscope. This fluorescent dye was excited at $365 \mathrm{~nm}$ and viewed through a 420-470 nm emission filter, and the photomicrographs of the stained cells were taken by color digital camera (AxioCam MRc 5, Carl Zeiss) driven by AxioVision 4.2 software (Carl Zeiss).

Survival rate of DRG neurons. DRG neurons were treated with 4-aminopyridine, 4-aminopyridine plus bafilomycin $\mathrm{A} 1$, or $\mathrm{NH}_{4} \mathrm{Cl}$, all dissolved in physiological salt solution, or with 4-aminopyridine in a $\mathrm{Cl}^{-}$-free solution. Control neurons were treated with physiological salt solution alone. The survival rate of these neurons was assayed just before and during a 24 hour treatment (one, two, three, four, five, and 24 hours after the start of treatment). The viability of cells was judged by trypan blue staining. The DRG neurons were stained with $0.1 \%$ trypan blue for one minute and washed with physiological salt solutions, and the stained dead neurons were observed under a microscope. Cell survival rate is expressed as mean \pm standard deviation (SD) of the percentage of live neurons relative to total neurons tested. The significance of differences among treatment groups at corresponding times was determined by repeated-measures analysis of variance (ANOVA) followed by a post hoc Bonferroni-Dunn test. Statistical significance was determined as $\mathrm{p}<0.01$.

\section{Results}

\section{Vacuole formation process}

High-resolution video-microscopy was used to observe in real time the cell structure changes (Figure 1). Cultured neuronal and non-neuronal cells derived from mouse dorsal root ganglia were treated with each of $10 \mathrm{mM} \mathrm{NH}_{4} \mathrm{Cl}, 4 \mathrm{mM}$ aminopyridines (2-aminopy-

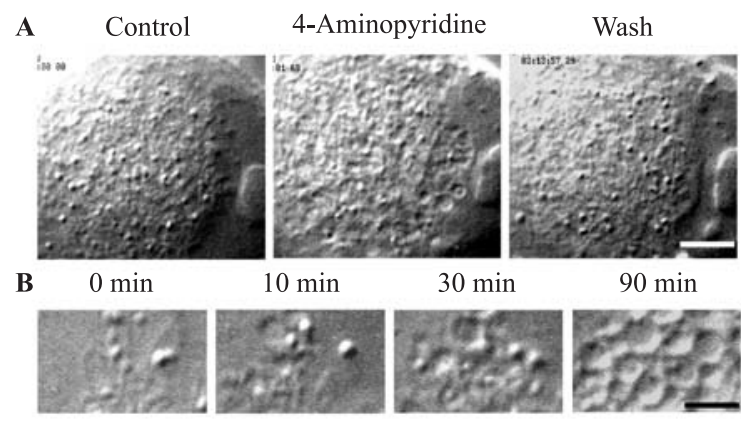

Figure 1. Reversible formation of cytoplasmic vacuoles around individual spherical organelles in mouse cells treated with weak base. A. Cell body of the dorsal root ganglion neuron before (control, left) and after one hour treatment with $4 \mathrm{mM}$ 4-aminopyridine (middle) and after two hour washout (right); B. Magnified images of vacuoles obtained before (0 minutes) and ten, 30, and 90 minutes after treatment with $4 \mathrm{mM}$ 4-aminopyridine in non-neuronal cells

ridine, 3-aminopyridine, 4-aminopyridine, and 3,4-diaminopyridine), and $50 \mu \mathrm{M}$ chloroquine. All these agents at these concentrations caused vacuolization within 30 minutes in all neuronal and non-neuronal cells. The results presented here were mostly obtained by using $4 \mathrm{mM} 4$-aminopyridine, but all these agents showed similar effects, whether they were strong or weak.

The process of vacuole formation was investigated on both neuronal and non-neuronal cells under video monitoring. The cells began to display small cavities around individual spherical organelles 5-10 minutes after the start of treatment with the weak base (Figure 1B). Simultaneously, the organelle showed Brownian movement inside the cavity. Thereafter, the cavities became larger, showing obvious vacuoles (diameter 1-10 $\mu \mathrm{m}$ ) 20-30 minutes after the start of treatment (Figure 1B), and the Brownian movement of the organelles within vacuoles gradually became more rapid.

Therefore, contents of vacuoles appear to be composed of serous fluid. The number of obvious vacuoles increased as time passed (Figure 1B). We never observed organelles swelling to become vacuoles. The size of organelles did not change throughout the vacuole formation period. Vacuoles were detected in all regions where spherical organelles were present in neurons (cell bodies, neurite shafts, and growth cones) and non-neuronal cells (perinuclear regions). These phenomena were reversed by washout with physiological salt solution for two hours after a one hour treatment with weak bases (Figure 1A): Vacuoles became smaller and the Brownian movement of organelles gradually diminished. However, longer treatment (three-24 hours) of weak bases resulted in cell 


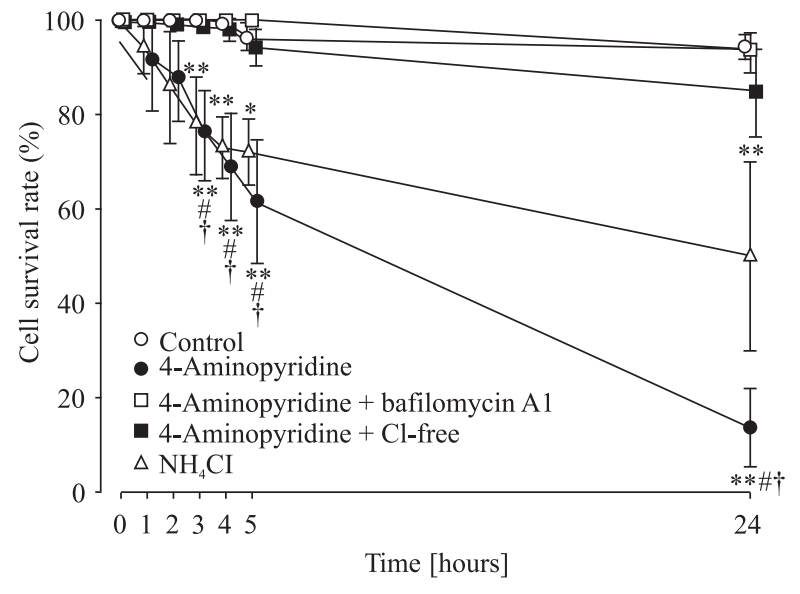

Figure 2. Survival rate of dorsal root ganglion neurons treated with weak base. Neurons were treated with physiological salt solution alone (control, open circles), $4 \mathrm{mM}$ 4-aminopyridine dissolved in physiological salt solution (solid circles), $4 \mathrm{mM} 4$-aminopyridine plus $1 \mu \mathrm{M}$ bafilomycin $\mathrm{A} 1$ in physiological salt solution (open squares), $4 \mathrm{mM}$ 4-aminopyridine in $\mathrm{Cl}^{-}$-free solution (solid squares), and $10 \mathrm{mM}$ ammonium chloride $\left(\mathrm{NH}_{4} \mathrm{Cl}\right)$ in physiological salt solution (open triangles). Each point indicates the mean. Error bars are standard deviations. $\mathrm{n}=5$ culture per group; ${ }^{*} \mathrm{p}<0.001 ; * * \mathrm{p}<0.0001$ compared to control; ${ }^{\#} \mathrm{p}<0.0001$ compared to 4-aminopyridine plus bafilomycin A1; ${ }^{\dagger} \mathrm{p}<0.0001$ compared to 4-aminopyridine in $\mathrm{Cl}^{-}$-free solution at corresponding time

death, which was estimated by trypan blue staining in neurons (non-neuronal cells were not tested, since they can proliferate, causing less accurate assay of cell death) (Figure 2). The intravacuolar organelles continued to move until the end of the experiments ( 24 hours after the start of treatment), even in dead cells.

\section{Dynamics of lysosomes, mitochondria, and actin filaments during vacuolization}

We monitored the dynamics of fluorescent protein-tagged lysosomes, mitochondria, and actin filaments after treatment with 4-aminopyridine $(4 \mathrm{mM})$ in living non-neuronal cells under confocal microscopy. The vacuole was confirmed to be formed around the lysosome detected by the expression of RFP-tagged lysosomal protein (Figure 3A). Mitochondria visualized by GFP were extruded outside the vacuoles and they comprised a part of the rim of vacuoles (Figure 3B). As shown in Figure 3C, after one hour of treatment with 4-aminopyridine, the filamentous structure of GFP-tagged actins was disrupted and the aggregation of actin filaments was increased at the vacuolation area. Actin filaments were not detected inside the vacuole.

\section{Cellular distribution of proteins/amino acids in vacuolated cells}

When living cells were stained with the cell-permeant amine-reactive fluorescence CFDA-SE, which labels cytoplasmic proteins and amino acids, the cytoplasm, but not the inside of vacuoles, was stained (Figure 4A). Thus, vacuoles are unlikely to contain proteins or amino acids.

\section{Internalization of weak base into organelles}

Neuronal and non-neuronal cells were treated with chloroquine $(50 \mu \mathrm{M})$ for one hour and then immunostained with anti-chloroquine antibody to investigate the cellular distribution of chloroquine under confocal microscopy. The organelles inside the vacuoles were immunostained with anti-chloroquine antibody (Figure 4B). This result confirmed that weak bases are internalized into organelles, as predicted theoretically [12]. The cytoplasm was also stained, but vacuoles except organelles were scarcely stained, indicating that the weak base also distributed in the cytoplasm, but not in the vacuoles.

\section{Acidity of organelles and vacuoles}

Living cells were stained with LysoTracker Green, an $\mathrm{H}^{+}$sensitive fluorescent probe that concentrates in acidic intracellular compartments. In control cells not treated with weak base, only some spherical organelles were stained (Figure 4C). When the LysoTracker-pre-loaded cells were then treated with $4 \mathrm{mM}$ 4-aminopyridine for one hour (pre-loaded LysoTracker), only intravacuolar organelles were stained (Figure 4D), indicating that vacuoles contain the organelles still acidic even under the vacuolated condition. When cells were treated with 4-aminopyridine for one hour and then loaded with LysoTracker Green (post-loaded Lysotracker), the organelles inside the vacuoles were strongly stained by LysoTracker, and, significantly, the vacuoles outside the organelles were slightly stained (Figure 4E). Observation of the cells that were treated with weak base and then loaded (post-loaded) with LysoSensor Yellow/Blue revealed that the intravacuolar organelles were stained yellow and vacuoles were stained blue (Figure 4F), indicating that organelles were strongly acidic and vacuoles were slightly acidic. The cytoplasm outside the vacuoles was not stained by these probes (Figures 4E, F). These observations, showing that vacuoles are weakly acidic, suggest that $\mathrm{H}^{+}$may be extruded from the organelle to its own vacuole during vacuolization. 


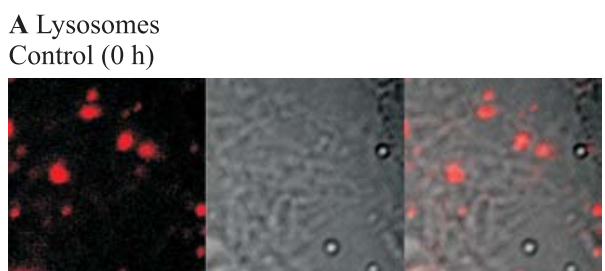

4-Aminopyridine (1 h)

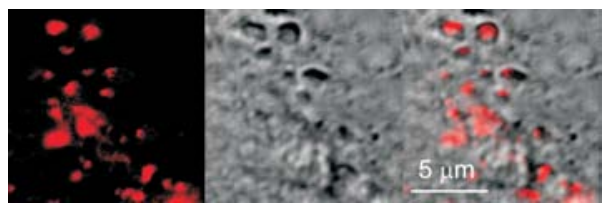

B Mitochondria

Control

4-Aminopyridine (1 h)
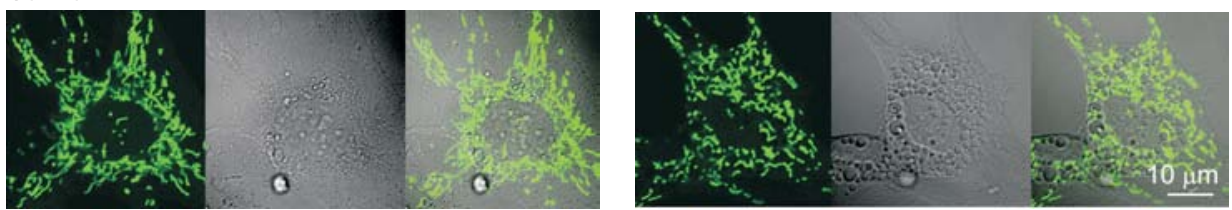

C Actin filaments

Control (0 h)

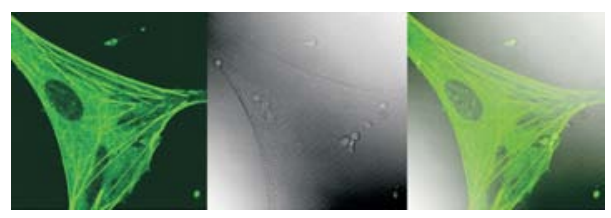

4-Aminopyridine (1 h)

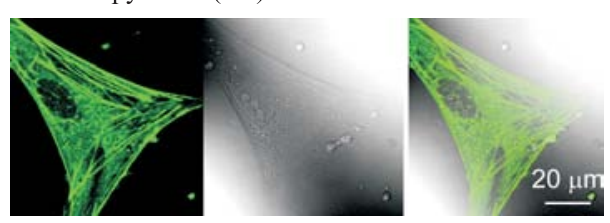

Figure 3. Fluorescent protein-tagged lysosomes, mitochondria, and actin filaments in vacuolated cells. Confocal microscopy images of RFP-labeled lysosomes (A), GFP-labeled mitochondria (B), and GFP-labeled actin filaments (C), before and one hour after treatment with $4 \mathrm{mM} 4$-aminopyridine. Left panels are fluorescence images, middle panels are differential interference contrast image, and right panels are merged images

\section{Independence of cathepsin $D$ from vacuole formation}

Cathepsin D is one of the lysosomal acid proteases activated under acidic conditions. Since the vacuole contents are likely to be serous fluid, it is possible that such proteases may leak out from the organelle to lyse the cytoplasm around acidic organelles to form vacuoles. Therefore we examined this possibility by observing the cellular distribution of cathepsin D immunoreactivity. However, the results contradicted our expectation: organelles were positively immunostained with anti-cathepsin $\mathrm{D}$ antibody, but the vacuoles were not stained (Figure 4G). Thus, we found no evidence that the formation of vacuoles is caused by the extrusion of acid protease from organelles. Since vacuole formation is reversible, molecules easily passing through the organelle membrane might be primarily involved in vacuole formation. Together with the knowledge that $\mathrm{H}^{+}$channels and cation $/ \mathrm{H}^{+}$exchanger, as well as vacuolar $\mathrm{H}^{+}$-ATPase, are expressed in acidic organelle membrane [13-16], we believe that $\mathrm{H}^{+}$can pass through the organelle membrane in both directions much more easily than protease.

\section{Involvement of vacuolar $\mathrm{H}^{+}$-ATPase and $\mathrm{Cr}$}

Vacuolar $\mathrm{H}^{+}$-ATPase is essential for vacuolization induced by weak bases [5], organic amines [17], and toxins of Helicobacter pylori $[18,19]$ and Vibrio cholerae [4]. We previously showed that 4-aminopyridineinduced vacuole formation associated with Brownian movement of organelles was blocked by vacuolar $\mathrm{H}^{+}$-ATPase inhibitor bafilomycin A1 [8].

As shown in Figure 5B, the present study confirms that the formation of vacuoles around acidic organelles was blocked by simultaneous treatment with $1 \mu \mathrm{M}$ bafilomycin A1. As shown in Figure 2, the survival rate of DRG neurons treated simultaneously with 4-aminopyridine $(4 \mathrm{mM})$ and bafilomycin A1 $(1 \mu \mathrm{M})$ was higher than that of neurons only treated with 4-aminopyridine three to 24 hours after the start of treatment, and not different from non-treated control neurons. Thus, the effects of weak bases are mediated through the activity of vacuolar $\mathrm{H}^{+}$-ATPase, and cell death is caused by long-lasting vacuolation, but not by the presence of weak bases per se.

The vacuolar $\mathrm{H}^{+}$-ATPase requires membrane-permeable anions such as $\mathrm{Cl}^{-}[16]$. We therefore investigated the requirement of extracellular $\mathrm{Cl}^{-}$. The effects of weak bases, i.e. vacuole formation, Brownian movement of intravacuolar organelles, and cell death, were blocked by the $\mathrm{Cl}^{-}$-free (replaced by non-membrane-permeable $\mathrm{SO}_{4}{ }^{2-}$ ) extracellular medium (Figures 2 and $5 \mathrm{C}$ ). These results suggest the requirement of extracellular $\mathrm{Cl}^{-}$for vacuole formation that leads to cell death. 

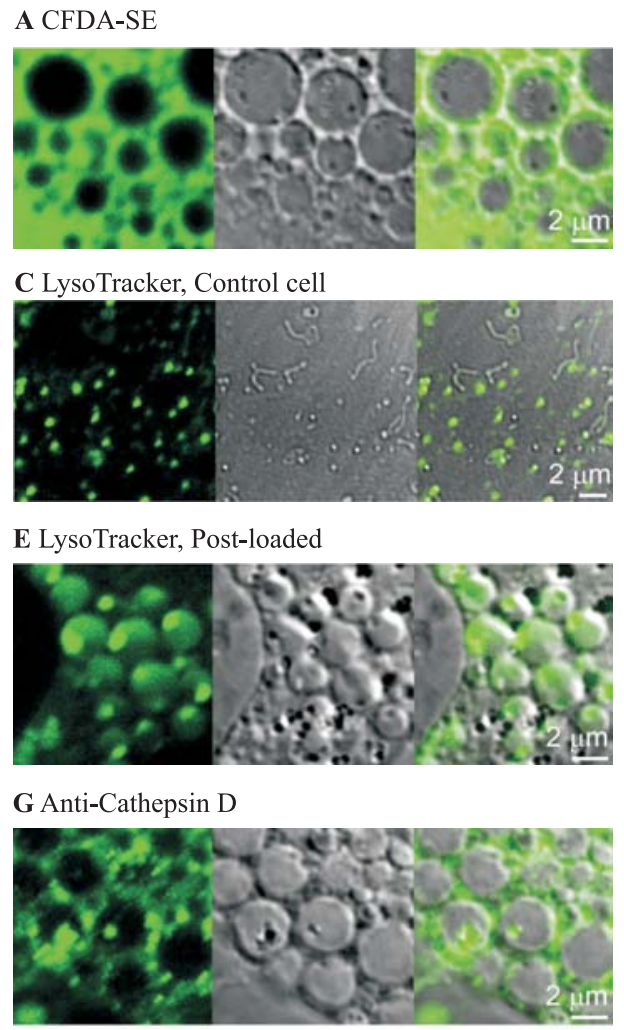

B Anti-Chloroquine

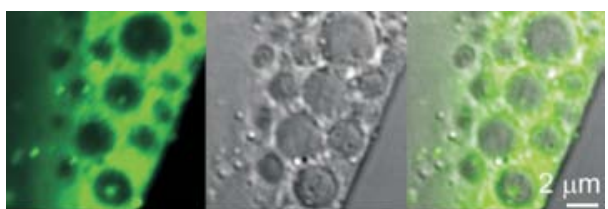

D LysoTracker, Pre-loaded

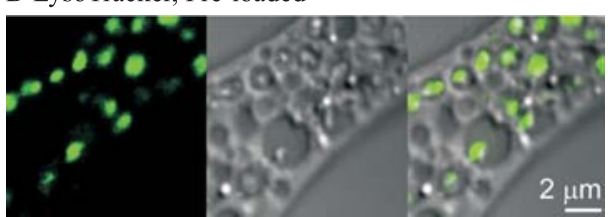

F LysoSensor Yellow/Blue

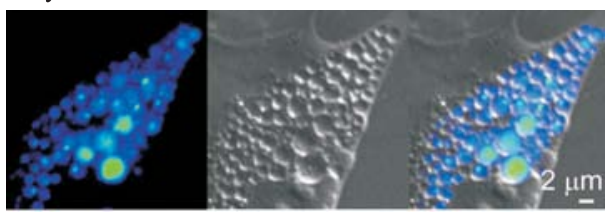

pH 47

Figure 4. Intracellular localization of protein or amino acids, the weak base, and $\mathrm{H}^{+}$in cells treated with weak bases. A. A non-neuronal cell treated with $4 \mathrm{mM} 4$-aminopyridine for one hour and then loaded with CFDA-SE, a membrane-permeant amine-reactive dye that labels proteins and amino acids. Note that organelles were not stained by CFDA-SE (either in 4-aminopyridine-treated cells or in control cells); B. A non-neuronal cell treated with chloroquine $(50 \mu \mathrm{M})$ for one hour was immunostained with anti-chloroquine antibody; C. An untreated non-neuronal cell (control) stained with LysoTracker Green, a fluorescent probe of cytoplasmic acidic compartments; D. A non-neuronal cell pre-loaded with LysoTracker Green and then treated with $4 \mathrm{mM}$ 4-aminopyridine for one hour. E. A non-neuronal cell treated with 4 mM 4-aminopyridine for one hour and then loaded with LysoTracker Green. F. A non-neuronal cell treated with $4 \mathrm{mM}$ 4-aminopyridine for one hour and then loaded with LysoSensor Yellow/Blue. G. A non-neuronal cell immunostained with anti-cathepsin D (a lysosomal acid protease) antibody. Left panels are fluorescence images, middle panels are differential interference contrast image, and right panels are merged images

\section{Discussion}

We have here demonstrated the formation process and characteristics of weak base-induced cytoplasmic vacuoles using neuronal and non-neuronal cells.

There was no essential difference between neuronal and non-neuronal cells. Video-microscopic observation showed that vacuoles are formed around individual spherical organelles. Biochemical study using low $\mathrm{pH}$ indicators and fluorescent protein-tagged lysosomal protein indicated that the intravacuolar organelles were confirmed to be acidic organelles such as lysosomes. Acidic organelles in the vacuoles showed Brownian movement, and the vacuoles did not contain proteins or amino acids. Thus, the vacuoles are likely to be serous liquid substances partitioned from the cytosol.

The fluorescent protein expression also showed that mitochondria and actin filaments composed part of the rim of vacuoles. These results are supported by a previous electron microscopic observation by Henics and Wheatley [20] which showed that the cytoplasmic vacuoles produced by the weak base procaine are membraneless. They proposed that the membraneless vacuoles are explained by a non-membrane-based phaseseparation of intracellular water. Perhaps, vacuolar contents with low viscosity are surrounded by the viscous cytosol, other organelles, and cytoskeletons, and the boundary between these regions is visible, like a membrane. However, in contrast to the present results, a study using isolated lysosomes suggested that vacuole formation by weakly basic substances is caused by osmotic uptake of water into organelles [6]. It has also theoretically suggested that the VacA-induced and vacuolar $\mathrm{H}^{+}$-ATPase-dependent vacuole formation is due to water influx from the cytosol to the lumen of late endosomes [21]. We sometimes observed spontaneously swollen spherical organelles, but the dif- 
$\mathbf{A}$

$\stackrel{\text { A }}{\text { 4-Aminopyridine }}$

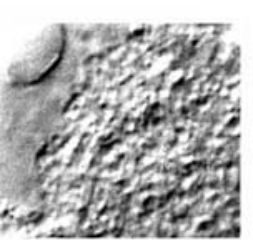

C

4-Aminopyridine in $\mathrm{Cl}^{-}$-free

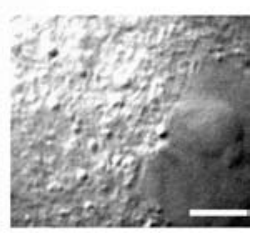

Figure 5. Role of vacuolar $\mathrm{H}^{+}$-ATPase and $\mathrm{Cl}^{-}$on vacuolization. Video-microscopic image of the cell body of dorsal root ganglion neuron treated for one hour with $4 \mathrm{mM}$ aminopyridine (A), with $4 \mathrm{mM}$ 4-aminopyridine plus $1 \mu \mathrm{M}$ bafilomycin $\mathrm{A} 1$ (B), and $4 \mathrm{mM} 4$-aminopyridine in $\mathrm{Cl}^{-}$-free solution $(\mathbf{C})$

ferential interference contrast (DIC) image of the weak base-induced vacuoles was clearly different from that of swollen organelles: the weak base-induced vacuoles were concave, whereas the swollen vacuoles were convex. In addition, we observed that the size of organelles did not change during a vacuole formation period. This underscores that weak base-induced vacuoles are not organelles themselves, but are formed around acidic organelles.

We also investigated mechanisms for vacuole formation induced by weak bases. The present cytochemical staining study indicated that the organelle inside the vacuole contains weak base and $\mathrm{H}^{+}$. Vacuolar $\mathrm{H}^{+}-$ -ATPase inhibitor bafilomycin $\mathrm{A} 1$ and $\mathrm{Cl}^{-}-$free medium blocked vacuole formation and subsequent cell death. These components have been demonstrated to be necessary for the vacuole formation induced by weak bases [6] and bacterial toxins, Helicobacter pylori toxin VacA [18, 19, 22] and Vibrio cholerae toxin [4]. The vacuolization induced by these toxins probably needs weak bases $[4,21]$. The weak bases are trapped in acidic organelles by intraorganelle protonation of the weak base [5, 23, 24]. Thus, weak bases can activate vacuolar $\mathrm{H}^{+}$-ATPase, allowing $\mathrm{H}^{+}$to enter the organelle and prevent the excessive increase in intraorganelle $\mathrm{pH}$. Activation of vacuolar $\mathrm{H}^{+}$-ATPase may, in turn, accelerate the trapping of weak bases. Thus, weak bases possibly accumulate within organelles until reaching the equilibrium, as suggested by de Duve et al. [12]. In association with this, water should be taken up into the organelles to keep intraorganelle osmolarity constant, and then water pressure inside the organelle increases [5, 6]. At this time, free $\mathrm{H}^{+}$should still present in the organelles [25]. Indeed, the present study using LysoTracker showed that intravacuolar organelles were still acidic after treatment with weak base. Significantly, the vacuoles outside the organelles were also slightly acidic. In addition, the contents of vacuoles are serous without proteins or amino acids and also without weak base. Thus, it is possible that vacuoles are formed by extrusion of $\mathrm{H}^{+}$from acidic organelles along with water. Further studies are needed to prove this.

The present observation indicated vacuole formation process and mechanism that may underlie the vacuolation observed in some pathological states, poisonings by weak base drugs such as aminopyridines and chloroquine.

\section{Acknowledgements}

This work was partially supported by a Grant-in-Aid for Scientific Research (C) (KAKENHI 14180001 from the Japan Society for the Promotion of Science (JSPS) to H.H.

\section{References}

1. Cover TL, Vaughn SG, Cao P, Blaser MJ. Potentiation of Helicobacter pylori vacuolating toxin activity by nicotine and other weak bases. J Infect Dis. 1974; 166:1073-1078.

2. Kourie JI. Prion channel proteins and their role in vacuolation and neurodegenerative diseases. Eur Biophys J. 2002; 31:409-416.

3. Méresse S, Steele-Mortimer O, Moreno E, Desjardins M, Finlay B, Gorvel JP. Controlling the maturation of pathogen-containing vacuoles: a matter of life and death. Nat Cell Biol. 1999;1:E183-E188.

4. Moschioni M, Tombola F, de Bernard M et al. The Vibrio cholerae haemolysin anion channel is required for cell vacuolation and death. Cell Microbiol. 2002;4:397-409.

5. Ohkuma S, Poole B. Cytoplasmic vacuolation of mouse peritoneal macrophages and the uptake into lysosomes of weakly basic substances. J Cell Biol. 1981;90:656-664.

6. Ohkuma S, Takano T. ATP-dependent lysis of isolated lysosomes by basic substances and acidic ionophores. Cell Struct Funct. 1997;22:253-268.

7. Fedorko ME, Hirsch JG, Cohn ZA. Autophagic vacuoles produced in vitro. I. Studies on cultured macrophages exposed to chloroquine. J Cell Biol. 1968;38:377-391.

8. Hiruma H, Kawakami T. Effects of 4-aminopyridine on organelle movement in cultured mouse dorsal root ganglion neurites. J Mol Neurosci. 2010 (in press).

9. Glover WE. The aminopyridines. Gen Pharmacol. 1982; 13: 259-285.

10. Schechter LE. The potassium channel blockers 4-aminopyridine and tetraethylammonium increase the spontaneous basal release of $\left[{ }^{3} \mathrm{H}\right] 5$-hydroxytryptamine in rat hippocampal slices. J Pharmacol Exp Ther. 1997;282: 262-270.

11. Nashmi R, Fehlings MG. Mechanisms of axonal dysfunction after spinal cord injury: with an emphasis on the role of voltage-gated potassium channels. Brain Res Brain Res Rev. 2001; 38:165-191.

12. de Duve C, de Barsy T, Poole B, Trouet A, Tulkens P, Van Hoof F. Commentary. Lysosomotropic agents. Biochem Pharmacol. 1992;23:2495-2531.

13. Beyenbach KW, Wieczorek H. The V-type $\mathrm{H}^{+}$ATPase: molecular structure and function, physiological roles and regulation. J Exp Biol. 2006;209:577-589. 
14. Moriyama Y, Maeda M, Futai M. Involvement of a non-proton pump factor (possibly Donnan-type equilibrium) in maintenance of an acidic pH in lysosomes. FEBS Lett. 1992; 302:18-20.

15. Nakamura N, Tanaka S, Teko Y, Mitsui K, Kanazawa H. Four $\mathrm{Na}^{+} / \mathrm{H}^{+}$exchanger isoforms are distributed to Golgi and post-Golgi compartments and are involved in organelle $\mathrm{pH}$ regulation. J Biol Chem. 2005;280:1561-1572.

16. Nishi T, Forgac $\mathrm{M}$. The vacuolar $\left(\mathrm{H}^{+}\right)$-ATPases: nature's most versatile proton pumps. Nat Rev Mol Cell Biol. 2002;3:94-103.

17. Morissette G, Moreau E, C-Gaudreault R, Marceau F. $\mathrm{N}$-Substituted 4-aminobenzamide (procaineamide analogs): an assessment of multiple cellular effects concerning ion trapping. Mol Pharmacol. 2005;68:1576-1589.

18. Cover TL, Reddy LY, Blaser MJ. Effects of ATPase inhibitors on the response of Hela cells to Helicobacter pylori vacuolating toxin. Infect Immun. 1993;61:1427-1431.

19. Papini E, Bugnoli M, De Bernard M, Figura N, Rappuoli R, Montecucco C. Bafilomycin A1 inhibits Helicobacter pyloriinduced vacuolization of HeLa cells. Mol Microbiol. 1993; $7: 323-327$.
20. Henics T, Wheatley DN. Vacuolar cytoplasmic phase separation in cultured mammalian cells involves the microfilament network and reduces motional properties of intracellular water. Int J Exp Pathol. 1997;78:343-354.

21. Tombola F, Carlesso C, Szabň I et al. Helicobacter pylori vacuolating toxin forms anion-selective channels in planar lipid bilayers: possible implications for the mechanism of cellular vacuolation. Biophys J. 1999;76:1401-1409.

22. Morbiato L, Tombola F, Campello S et al. Vacuolation induced by VacA toxin of Helicobacter pylori requires the intracellular accumulation of membrane-permeant bases, $\mathrm{Cl}^{-}$ and water. FEBS Lett. 2001;508:479-483.

23. Arai K, Yasuda N, Isohashi F, Okamoto K, Ohkuma S. Inhibition of weak-base amine-induced lysis of lysosomes by cytosol. J Biochem. 2002;132:529-534.

24. Wada Y, Ohsumi Y, Anraku Y. Chloride transport of yeast vacuolar membrane vesicles: a study of in vitro vacuolar acidification. Biochim Biophys Acta. 1992;1101:296-302.

25. Poole B, Ohkuma S. Effect of weak bases on the intralysosomal $\mathrm{pH}$ in mouse peritoneal macrophages. $J$ Cell Biol. 1981;90:665-669. 